\title{
Study of Reliability in a Repairable System by Markov Chains
}

\author{
Naima TAMALOUSSI ${ }^{1}$, Azzedine BOUZAOUIT ${ }^{2}$ \\ ${ }^{1}$ Department of Mechanical Engineering, Faculty of Technology, University of Skikda, Algeria, \\ e-mail: naimatamaloussi@yahoo.com \\ ${ }^{2}$ Department of Mechanical Engineering, Faculty of Technology, LRPCSI Laboratory of \\ research, University of Skikda, Algeria, e-mail: bouzaouit21@gmail.com
}

Manuscript received November 15, 2019; revised November 11, 2020.

\begin{abstract}
In the industrial sector, maintenance of production facilities plays an important role to carry out production by increasing the reliability and availability of the production process. Predictive maintenance strategy seems adequate to anticipate the failure and degradation of the state of such equipment. A reliability study is needed to quantify indicators to describe the functioning of any system over time. In this paper, we present the results of a stochastic modeling conducted on the analysis of the availability of motor-pump system, installed in a cooling circuit in an industrial complex. The equipment considered in this study is composed of four subsystems. The proposed model is a dynamic Markovian approach, for the purpose of a comparison with the analytical calculation in terms of the indicators' evaluation of the dependability of the studied system, including instant availability. The different states of the system components and the transition functions between these states have also been characterized. The results of availability obtained by the model are well correlated with those calculated analytically, confirming that the proposed model is very powerful, it will help predict the future states of the system, in order to predict any necessary preventive maintenance actions.
\end{abstract}

Keywords: Reliability, repairable system, Markov chain, modelling, transition matrix.

\section{Introduction}

The reliability of the various industrial achievements becomes an important issue at the economic, ecological and human levels. Technology is moving too fast, materials are becoming too complex for common sense to be enough to predict the risks [1], [2]. Stochastic processes such as the Markov processes are 
used to model the evolution of a system in the course of time [3]. The quantification of these processes is carried out either by analytical calculations when that is possible, or using simulation of Assembles-Carlo, or with deterministic numerical algorithms [4]. These tools of quantification can be difficult to use for people not initiated with these methods, thus tools of assistance to modeling such as the Dynamic Bayesian Networks, the Boolean logic driven Markov processes BDMP, were developed. These tools are generally coupled with the calculation algorithms. The quantification of the systems to be modeled is assisted by an intuitive chart of the system.

For a whole reparable system, adequate models are required to ensure a good risk prediction, so to have a good performance of the system. This modeling is called stochastic modeling.

The stochastic modeling often relates to reparable systems. This approach is the base of the monitoring methods, diagnosis and maintenance.

For a reparable system, it is necessary to introduce another probabilistic concept: the availability $A(t)$ is the probability that the device functions at moment $t[5]$.

For that, one can rely on the description of the different degradations states of the system until the failure. The passage from a state to another is done by transitions. These transitions are regarded as instantaneous in the Markov model [6]. A stochastic process is a whole of random variables $\left(X_{T}\right)_{T=0}$ with values in the whole of the observations. A process is Markovian if the transition probability of the future states depends on the present state but does not depend on the past states [7].

The stochastic processes are used in modeling systems of discrete nature, and more precisely to model the temporal evolution of the dynamic systems. Indeed, such systems generally evolve from one state to another, and what matters to the modeler is the characterization of these states changes. Both the discrete-time and the continuous-time Markov chains [8], are defined and characterized by their probabilities of transition from a state to another [9].

The Markov chains are a succession of random variables $\left(X_{n}, n \in N\right)$, where $X_{n}$ represents the state of the system at moment $n$. The fundamental property of Markov chains known as property of Markov is that its future evolution depends on the past only through its current value. In other words, conditionally with $X_{n}$ and $X_{n+k}(k \in N)$ are independent. The applications of the Markov chains [10] are very numerous (networks, genetics of the populations, mathematical, stock management, stochastic algorithms of optimization, simulation).

We can then define the law of "probability of transition" from a state (i) towards another state $(j)$ by:

$$
P_{i, j}=P\left(X_{n}=j \mid X_{n-1}=i\right) .
$$


A system can be defined like a whole of interdependent components, designed to fulfill a given function, under conditions given at an interval of time [11].

For each system, it is important to clearly define the elements, which characterize it, namely: the function, the structure, the operating conditions and the environment in which it has to operate. The reliability of a system is expressed by the probability that this device achieves a necessary function under conditions of use for a period of time; it is thus a value ranging between 0 and 1 . In the following we denote it by $R(t)$, where $t$ indicates the duration of the mission.

$$
R(t)=P\{\text { lifespan of the system }>t\} .
$$

Let us recall that the lifespan of a system is a measure of the rendered quantity of service. According to the studied system, it is expressed in term of time, kilometers, or other.

The fact that the failure of a system can occur at any time brings us to consider this quantity as a random variable to which we can associate a function of density of probability $f(t)$. It is important to recall that $f(t)$ is the probability that the lifespan of a system lies between $t$ and $t+d t$, or the probability that it breaks down between $t$ and $t+d t$.

$$
f(t) \cdot d t=P\{t<\text { lifespan of the system }<t+d t\} .
$$

The function of distribution or the function of distribution associated with the lifespan $F(t)$, can be interpreted as the probability that the system still functions at the moment $t$.

$$
F(t)=P\{\text { lifespan of the system } \leq t\} .
$$

The failure of equipment can be characterized by a rate $(\lambda)$ called failure rate. This rate is also called rate of chance or death rate. It is defined as being the conditional probability that the equipment breaks down between the moment $t$ and $t+\Delta \mathrm{t}$ knowing that it survived until the moment $t$. It can also be defined as the proportion of components having survived until the moment $t$. It also represents the speed of arrival of the breakdown [12].

$$
\lambda(t)=\frac{N(t+\Delta t)-N(t)}{N(t)},
$$

with:

- $N(t)$ : component count having survived until the moment $t$;

- $N(t+\Delta t)$ : component count having survived until the moment $t+\Delta t$.

The increase in the complexity of a mechanical system makes its reliability to decrease, if one does not take compensatory measures. This system is formed of independent components that are to say in series. It is said that the system is without redundancy. The reliability of these systems worsens dramatically with the increase of the number of the components. 
When reliability is studied, one is interested not only in the probability of breakdown, but also in the number of breakdowns in particular, in order to repair the system on time.

From this point of view, two new parameters of reliability become our center of interest. The availability by definition is very close to reliability, except that the required system must function at the moment $(t)$ and not over a period of time $(0$ to $t)$.

For a system, with the assumption of a constant failure rate $\lambda$ and of a constant repair rate $\mu$, it is shown that the instantaneous availability is defined by (8):

$$
\begin{aligned}
\lambda & =1 / M T B F \\
\mu & =1 / M T T R,
\end{aligned}
$$

with:

$M T B F$ : Mean time between failures;

$M T T R$ : Mean time to restore.

The traditional instantaneous availability of the equipment is given by the following equation:

$$
A(t)=\frac{\mu}{(\lambda+\mu)}+\frac{\lambda}{\lambda+\mu} e^{-(\lambda+\mu) t} .
$$

When $\lambda$ and $\mu$ are independent of time, and when $t$ becomes large, one notes that $A(t)$ tends towards a constant value. This value is often called asymptotic availability. It is equal to:

$$
A_{\infty}=\frac{1}{1+\lambda / \mu} .
$$

The determination of $A(t)$ will be done starting from the estimate of the reliability parameters. By analogy with reliability, it is possible to give to maintainability a probabilistic definition: "maintainability is the probability of restoring a system under specified operating conditions, in wished limits of time, when maintenance is accomplished under conditions and with prescribed means" [12].

The maintainability index $(M)$ is the probability that a device is repaired before time $t$ :

$$
\begin{gathered}
M(t)=1-e^{-\mu t} \\
M T T R=\Sigma T R / N
\end{gathered}
$$

$M T T R$ : Mean time to repair;

$\sum T R$ : Total repair time. 


\section{Methodology of work}

We present in our study the case of a continuous process of feeding a boiler which feeds a steam turbine, which functions without interruption. The machine, which ensures the correct operation of this system, is a group motor-driven pump HPT 28-20, as can be seen in Fig. 1.

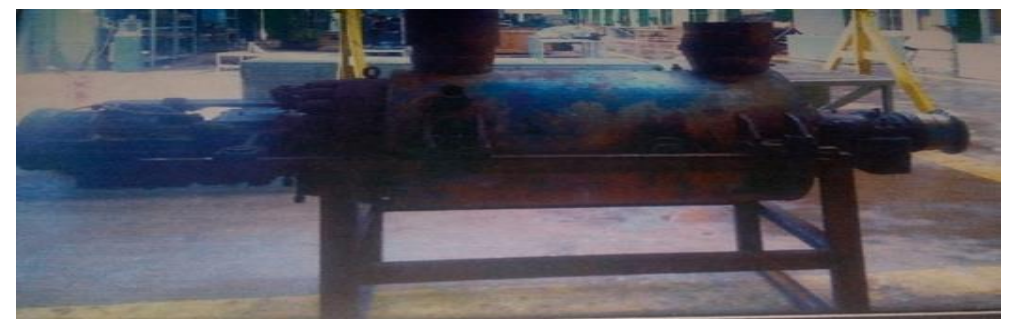

Figure 1: General view of the pump HPT 28-20 m 8 floors

We note that the group is made up of the following elements:

- an electric motor;

- a reducing coupler;

- a nourricier pump;

- a feeder pump.

\section{A. Determination of the reliability characteristics}

The machine history of failures and their times duration are summarized and given by Table 1 .

\section{Calculation of the failure rate $(\lambda)$}

To calculate the failure rate $\lambda$, we must calculate first the MTBF (Mean time between failure, equal: the sum of $T B F$ on $N$ ). In our case, the sum of $T B F$ is 29304 hours and $N$ is equal to 30 . Then we can calculate $\lambda$ (see (6)), TBF values are given in Table 1.

\section{Calculation of the repair rate $(\mu)$}

To calculate the rate of repair $\mu$ one must initially calculate the MTTR (Mean time to repair, equal: the sum of TTR on $N$ ). In our case, the sum of the TTR is 172 hours and $N$ is equal to 30 . Then one can calculate $\mu$ (see (7)). The values of TTR are given in Table 1. 
Table 1: Duration of good operation (TBF), and repair (TTR) of the feeder pump

\begin{tabular}{|c|c|c|c|c|c|c|c|}
\hline $\mathrm{N}^{\circ}$ & TTR(h) & TTF(h) & TBF(h) & $\mathrm{N}^{\circ}$ & TTR(h) & TTF(h) & TBF(h) \\
\hline 1 & 04 & 24 & 20 & 16 & 08 & 1104 & 1096 \\
\hline 2 & 08 & 1056 & 1048 & 17 & 08 & 600 & 592 \\
\hline 3 & 08 & 4296 & 4288 & 18 & 04 & 432 & 428 \\
\hline 4 & 18 & 1032 & 1014 & 19 & 04 & 912 & 908 \\
\hline 5 & 04 & 1896 & 1892 & 20 & 04 & 600 & 596 \\
\hline 6 & 08 & 168 & 160 & 21 & 18 & 576 & 558 \\
\hline 7 & 18 & 1776 & 1758 & 22 & 04 & 72 & 68 \\
\hline 8 & 01 & 648 & 647 & 23 & 15 & 672 & 457 \\
\hline 9 & 08 & 1344 & 1336 & 24 & 04 & 432 & 428 \\
\hline 10 & 08 & 1104 & 1096 & 25 & 01 & 312 & 311 \\
\hline 11 & 18 & 96 & 78 & 26 & 09 & 624 & 615 \\
\hline 12 & 16 & 3456 & 3440 & 27 & 08 & 336 & 328 \\
\hline 13 & 18 & 1704 & 1686 & 28 & 16 & 456 & 440 \\
\hline 14 & 08 & 1632 & 1624 & 29 & 16 & 96 & 80 \\
\hline 15 & 04 & 1272 & 1268 & 30 & 10 & 5520 & 5510 \\
\hline
\end{tabular}

The notation used in Table 1 is:

TTR: total repair time;

$T T F$ : total operating time;

$T B F$ : running time.

\section{B. Markov chain of the studied system}

This method results from a mathematical theory. One has a whole of states of components, which, combined, makes it possible to define the whole of the states of the system. This system is divided into two subsets:

- operating condition;

- state of breakdown.

One models the process like two subsystems $(S 1, S 2)$ "in series" (Sealing system - valve of safety). The breakdown of one of the two systems causes the breakdown of the system.

The Markov chain of the system is represented in Fig. 2. 


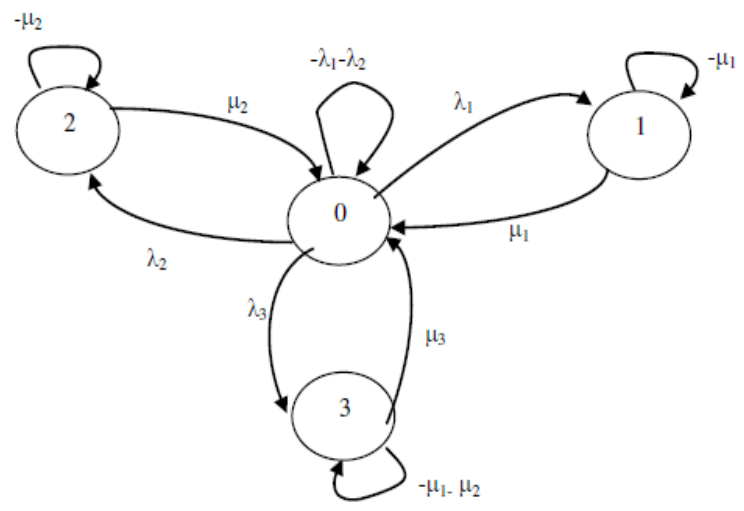

Figure 2: Markov graphical model of the system

The exponential law is selected to model the probability of failure of the feeder pump. The states of the system must be defined first. In the case of $N$ "binary" components, we get a maximum number of $2^{\mathrm{N}}$ states [13]. Then, it is necessary to count all the possible "transitions" between the various states from the system by identifying their causes. In our case $N=2$ thus the number of states is $2^{2}=4$ :

- State 0: S1, S2 subsystems function;

- State 1: $S 1$ subsystem is broken down;

- State 2: $S 2$ subsystem is broken down;

- State 3: subsystems $S 1, S 2$ are broken down, with:

S1: subsystem 1 (sealing system);

S2: subsystem 2 (valve of safety).

The associated equation of states is then:

$$
\begin{gathered}
P^{\prime}{ }_{0}(t)=-2\left(\lambda_{1}+\lambda_{2}\right) P_{0}(t)+\mu_{1} P_{1}(t)+\mu_{2} P_{2}(t)+\left(\mu_{1}+\mu_{2}\right) P_{3}(t) \\
P^{\prime}{ }_{1}(t)=\lambda_{1} P_{0}(t)-\mu_{1} P_{1}(t) \\
P^{\prime}{ }_{2}(t)=\lambda_{2} P_{0}(t)-\mu_{2} P_{2}(t) \\
P^{\prime}{ }_{3}(t)=\left(\lambda_{1}+\lambda_{2}\right) P_{0}(t)-\left(\mu_{1}+\mu_{2}\right) P_{3}(t) \\
A(S)=P_{0} \cdot(S I-M)^{-1}
\end{gathered}
$$

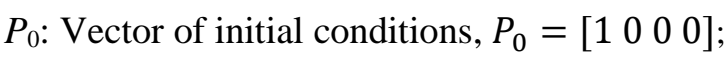

$P_{1}$ : Probability of state 1 ;

$S$ : Laplace variable;

$M$ : Transition matrix; 
I: Identity matrix, $I=\left[\begin{array}{cccc}1 & 0 & 0 & 0 \\ 0 & 1 & 0 & 0 \\ 0 & 0 & 1 & 0 \\ 0 & 0 & 0 & 1\end{array}\right]$.

The transition matrix $\mathrm{M}$ combines the graph:

$$
M=\left[\begin{array}{cccc}
-4 \lambda & \lambda & \lambda & 2 \lambda \\
\mu & -\mu & 0 & 0 \\
\mu & 0 & -\mu & 0 \\
2 \mu & 0 & 0 & -2 \mu
\end{array}\right] .
$$

Put: $\lambda_{1}=\lambda_{2}=\lambda_{3}=\lambda, \mu_{1}=\mu_{2}=\mu_{3}=\mu$.

The solution is based directly on the Laplace transform so the equation (16) becomes:

$$
A(S)=P_{0} \cdot\left[\begin{array}{cccc}
S+4 \lambda & -\lambda & -\lambda & -2 \lambda \\
-\mu & S+\mu & 0 & 0 \\
-\mu & 0 & S+\mu & 0 \\
-2 \mu & 0 & 0 & S+2 \mu
\end{array}\right]^{-1}
$$

The following model shows the availability of the system:

$$
A(s)=\frac{S^{2}+3 \mu S+2 \mu^{2}}{S^{3}+(4 \lambda+3 \mu) S^{2}+\left(6 \lambda \mu+2 \mu^{2}\right) S} .
$$

By returning to the time domain:

$$
A(t)=\frac{\mu}{3 \lambda+\mu}+\frac{6 \lambda\left(16 \lambda^{2}+\mu^{2}\right)}{2(3 \lambda+\mu)\left(16 \lambda^{2}+\mu^{2}\right)} \cdot e^{-\left(2 \lambda+\frac{3 \mu}{2}-\frac{1}{2} \sqrt{\left(16 \lambda^{2}+\mu^{2}\right)}\right) t} .
$$

\section{Results and discussion}

We have all the parameters necessary to plot the curves for the reliability and maintainability functions. They are represented respectively by Fig. 3 and Fig. 4 . 


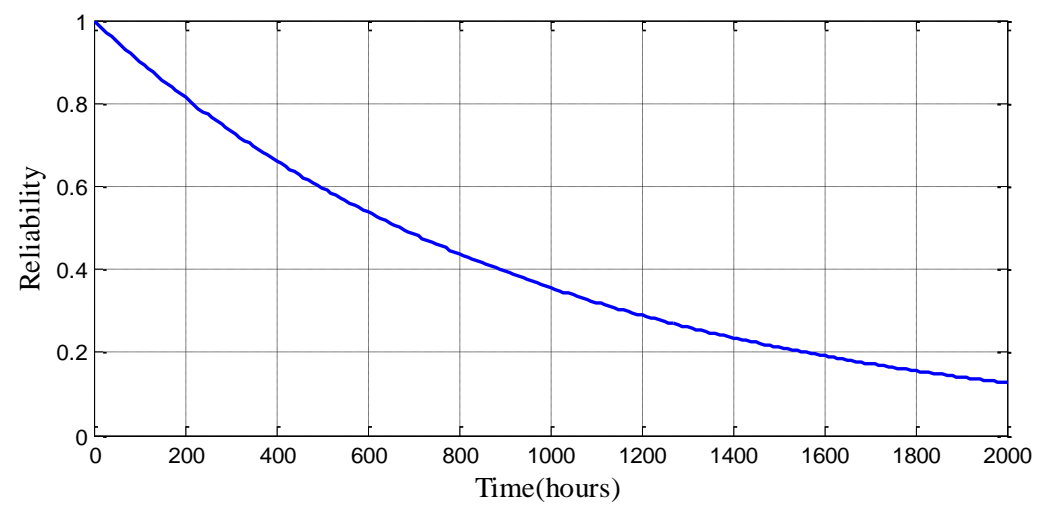

Figure 3: Curve of reliability

According to Fig. 3, which represents the reliability of the system, one can see clearly that for an average time of operation $(M T B F=976.8 \mathrm{~h})$, the reliability is about $37 \%$. This percentage of reliability is considered to be low, which can be interpreted as the absence of a regular maintenance program.

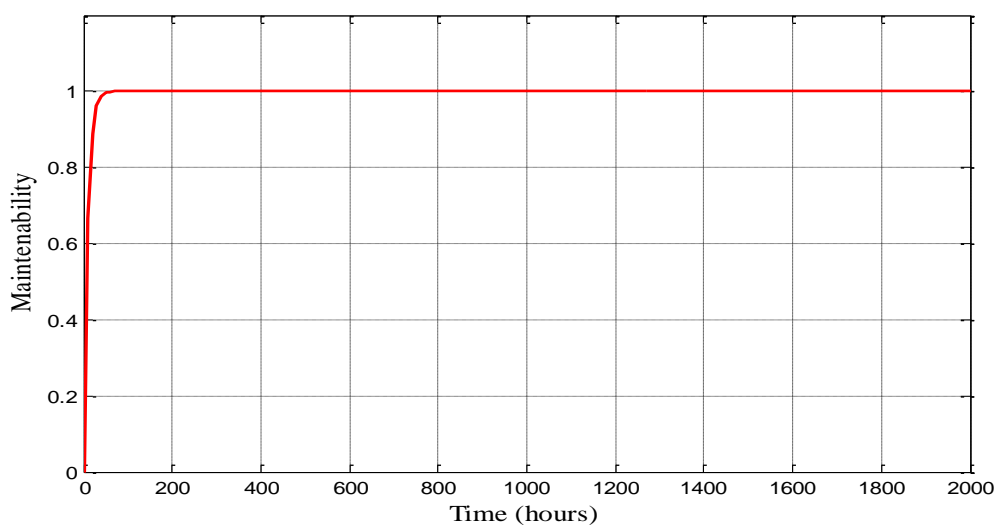

Figure 4: Curve of maintainability.

The maintainability of the motor-driven pump is represented in Fig. 4. According to this curve, one can see clearly that maintainability increases abruptly and reaches significant values in a very short time, the only interpretation of this being that the equipment encounters several technical problems, which require the stops to repair them. 
The availability is represented by Fig. 5 .

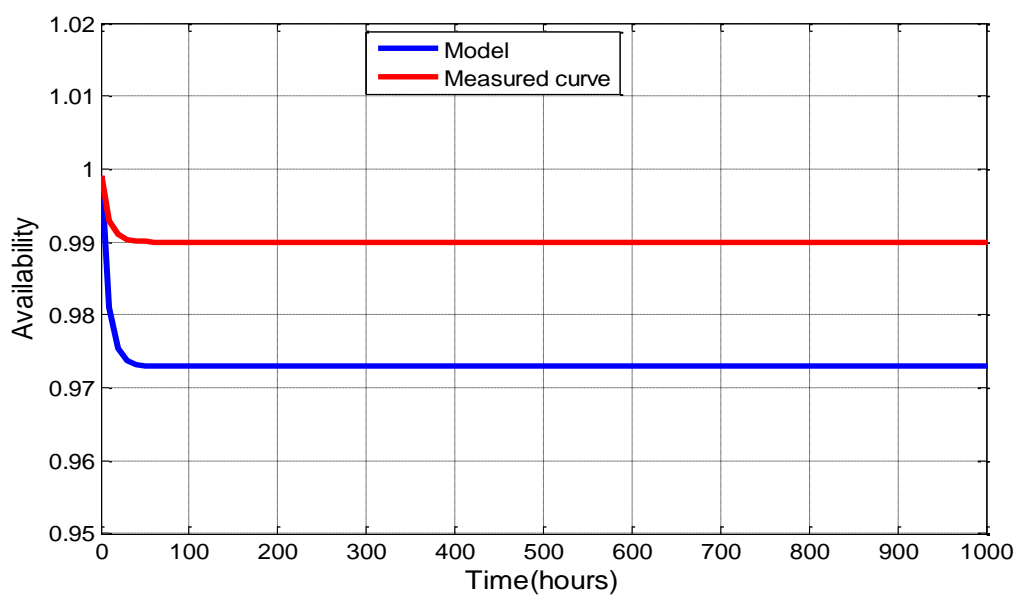

Figure 5: Curve of availability

Fig. 5 represents the availability of the system, according to analytical computations based on the $M T B F$ and according to the Markov model proposed; one can observe that the computed values and the model availability are well correlated. The coefficient of correlation is of the order $(R=0.996)$ this value confirms that the suggested Markov model is very powerful, and will contribute to the future prediction of the state of the motor-driven pump without waiting the breakdown. According to the objectives of the maintenance service (the availability of the system), and based on the proposed model, we can foresee the time necessary to schedule a preventive maintenance action.

\section{Conclusion}

Industrial systems are becoming complex and associated maintenance is becoming more expensive. Maintenance strategies are at the heart of this concern. These need to have reliability indicators, specific to each system that can be measured or modeled to trigger conditional preventive maintenance actions. On the other hand, predictive maintenance consists of using models to predict the reliability of the components of such a system, with the aim of increasing its availability through the implementation of anticipated interventions, in order to reduce the time required for maintenance. In this context, stochastic models of degradation are mathematical models that describe the degradation of a system over time. This degradation often leads to a decrease in the performance of the production system. Markov model is often used when evaluating the availability 
of repairable systems, based on the principle of changing the state of one component to another with the characterization of the transition between these states.

The proposed model is a dynamic Markov model approach, for the purpose of a comparison with the analytical calculation in terms of evaluation of availability indicator of the studied system. The different states of system components as well as the transition functions between these states have also been characterized. The proposed model adequately describes the instantaneous availability required for the future states prediction of the studied system, and consequently the improvement of the maintenance program.

\section{References}

[1] Alain, P., Michel, G., Maurice, M., "Reliability of systems", Editions Eyrolles, Paris, vol (15), No 4, pp. 778-790, Jul 2000.

[2] Jean, L., G., "Reliability of systems. Mathematical methods", Masson, Paris, 1991.

[3] Shophie, M., Cocozza, T., Michel, R., "Various stochastic models for maintenance optimization”, Journal of the French Statistical Society, vol 3, Paris, pp. 9-21, 2000.

[4] Cocozza, T., "Stochastic process and reliability of mathematical systems and applications", $\mathrm{N}^{\circ} 28,1997$.

[5] Pascal, "Modelling malfunctions of a system under maintenance activities. Research master's report", 2007.

[6] Agnes, L., Claudie, C., "Stochastic processes and modeling", ismag master 2 - mi00451x, 2012.

[7] Claire, P. E., "Dependability", $3^{\text {rd }}$ TR - SE option, 2012.

[8] Banege, L., "Stochastic process. Markov chains and Poisson Process", 2004.

[9] Bouzaouit, A., HadjadjAouel, E., Bennis, O. "Stochastic modeling for the follow-up the bearing degradation”, U.P.B, Sci Bull, N79, pp. 209-218, 2017.

[10] Bouzaouit, A., Bennis, O., Gahgah, M., "Modelling of the dynamic evolution of the state change of the bearings", RECENT Journal, vol (3), pp. 254-258, 2012.

[11] Parick, L., Marc, T., Toscana, R., "Reliability, diagnosis and predictive maintenance systems", Lavoisier SAS, 2012.

[12] Monchy, F., "The maintenance function, Training in Management of Industrial Maintenance", Collection of technologies from University to industry, MASSON, 1991.

[13] Benchouia, N., "The sacking of Reliability Systems has PEMFC fuel", Doctoral thesis, Skikda University, Algeria, 2014. 